\title{
Discovering intermediate massive black holes through tidally disrupted stars*
}

\author{
Martina Toscani ${ }^{\dagger}$ and Giuseppe Lodato ${ }^{\ddagger}$ \\ Department of Physics, University of Milan, \\ Celoria 16, Milan 20133, Italy \\ ${ }^{\dagger}$ martina.toscani@unimi.it \\ ¥giuseppe.lodato@unimi.it \\ Elena Maria Rossi \\ Leiden Observatory, J. H. Oort Building, Niels Bohrweg 2, \\ NL-2333 CA Leiden, The Netherlands \\ emr@strw.leidenuniv.nl
}

Received 11 June 2019

Accepted 21 August 2019

Published 15 October 2019

\begin{abstract}
Stars are spheres of gas held together by self-gravity. When flying by a black hole, however, the star self-binding force can be overwhelmed by the black hole tides and the star can be torn apart. This is a physically rich and fascinating event which will be described by first introducing the concept of black hole from a mathematical point of view. We will then dive into the physics of the tidal disruption and proceed describing the accompanying electromagnetic flare and gravitational wave burst in the frequency range of the Laser Interferometer Space Antenna. This empowers such events to discover the elusive black holes with mass intermediate between the solar and the million/billion solar masses.
\end{abstract}

Keywords: Gravitational waves; accretion; accretion discs; hydrodynamics; black hole physics.

The year was 1916 when a theoretical physicist, in his late thirties, published a theory destined to change the way we see and study the universe: this theory was general relativity (GR) and the man, it goes without saying, was Albert Einstein. At that time Einstein was already a renowned scientist, in particular thanks to the four articles published in 1905 (Einstein's annus mirabilis). However, GR is considered as Einstein's most important work, that made him known

\footnotetext{
*This essay received an Honorable Mention in the 2019 Essay Competition of the Gravity Research
} Foundation. 
also outside the scientific world. The vast impact that this theory had was in particular due to the fact that it provided a revolutionary description of gravity, that strides away from the classical concept introduced by Sir Isaac Newton of a force acting instantaneously between bodies. With GR, Einstein introduced the idea of gravity as a pure geometric feature, related to the curvature of spacetime.

Over the years, numerous experiments have confirmed the predictions of GR. In particular, one of the most important confirmations of the theory has been the detection of gravitational waves (GWs), occurred on 14th of September 2015, when the Laser Interferometer Gravitational-wave Observatory (LIGO) revealed the merger of two stellar black holes $(\mathrm{BHs})$ through the gravitational signal. This detection marked the beginning of a new era in astronomy: GW astronomy.

In this paper, we are interested in illustrating how, thanks to GW astronomy together with the study of the electromagnetic signal, it might be finally possible to detect a type of objects, allowed by GR, but not observed yet: intermediate massive black holes (IMBHs), with mass intermediate between the solar mass type observed in GWs and the million/billion solar mass BHs observed electromagnetically at the center of galaxies. In particular, we start giving a formal definition of $\mathrm{BHs}$, considering the solution of Einstein's equations in vacuum, with spherical symmetry. Then, we introduce an astrophysical phenomenon that involves BHs, that is tidal disruption events (TDEs). Eventually, we show how these events could be crucial to reveal IMBHs.

To introduce the concept of (nonrotating) BH, we consider the solution of Einstein's field equations in vacuum, under spherical symmetry, that is given by the Schwarzschild metric 1

$$
d s_{\mathrm{s}}^{2}=-\left(1-\frac{r_{\mathrm{s}}}{r}\right) c^{2} d t^{2}+\left(1-\frac{r_{\mathrm{s}}}{r}\right)^{-1} d r^{2}+r^{2}\left(d \theta^{2}+\sin ^{2} \theta d \phi^{2}\right)
$$

where $(t, r, \theta, \phi)$ are the Schwarzschild coordinates, $c$ is the speed of light and $r_{\mathrm{s}}$ is the Schwarzschild radius, defined as

$$
r_{\mathrm{s}} \doteq \frac{2 G M}{c^{2}}
$$

with $M$ being the mass sourcing the deformation and $G$ the gravitational constant. $r_{\mathrm{s}}$, for an object like the Sun, is about $3 \mathrm{~km}$. It is easy to see from Eq. (1), that this metric has two singularities: one at $r=0$ and one at $r=r_{\mathrm{s}}$. The first is an intrinsic singularity, which tells us that the curvature of the spacetime becomes divergent at this point. The second may be eliminated by a change of the coordinate system, thus it is not a singularity of spacetime itself rather than a coordinate singularity. However, it has an interesting physical meaning, that we can easily visualize in the following way. We rewrite Eq. (1) in terms of the Kruskal coordinates $(T, X)$, 


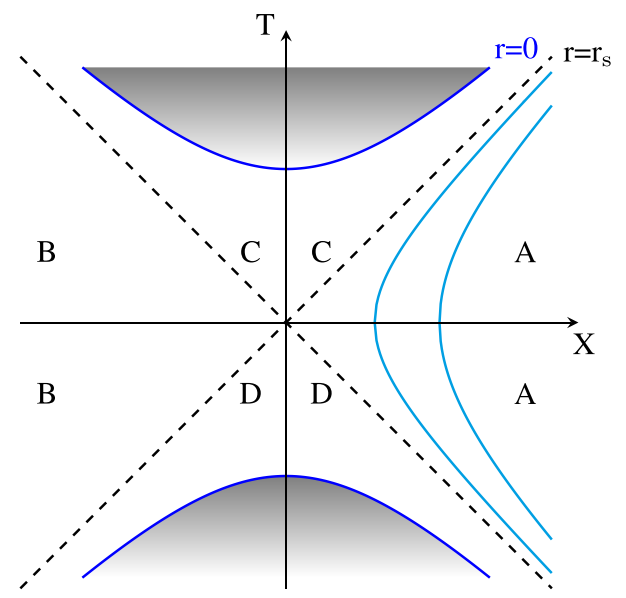

Fig. 1. (Color online) Kruskal diagram of the Schwarzschild spacetime. We identify four regions, $\mathrm{A}$ and $\mathrm{B}$, that lie outside the event horizon $\left(r=r_{\mathrm{s}}\right.$, dashed black lines), $\mathrm{C}$, identified as the $\mathrm{BH}$ and D, which is the white hole. The origin is the wormhole. The blue hyperbolas indicate the intrinsic singularity $(r=0)$, the cyan curves are geodesics of uniformly accelerated particles.

defined as

$$
\begin{aligned}
\left(\frac{r}{r_{\mathrm{s}}}-1\right) e^{\left(r / r_{\mathrm{s}}\right)} & =X^{2}-T^{2}, \\
\frac{c t}{r_{\mathrm{s}}} & =2 \tanh ^{-1} \frac{T}{X},
\end{aligned}
$$

in which the metric reads

$$
d s_{\mathrm{k}}=\frac{4 r_{\mathrm{s}}^{3}}{r} e^{-r / r_{\mathrm{s}}}\left(-d T^{2}+d X^{2}\right)+r^{2}\left(d \theta^{2}+\sin ^{2} \theta d \phi^{2}\right) .
$$

If we plot Eq. (3) in the $X-T$ plane, as done in Fig. 1. we identify four different regions. A and $\mathrm{B}$ are the portions of spacetime lying outside the coordinate singularity $\left(r>r_{\mathrm{s}}\right)$. The cyan curves in these regions are geodesics of uniformly accelerated particles. $\mathrm{C}$ and $\mathrm{D}$ have $r_{\mathrm{s}}>r>0$, while the blue hyperbolas indicate the intrinsic singularity $r=0$. Every particle or light ray in $\mathrm{C}$ will reach the singularity $r=0$ for $T \rightarrow+\infty$. In other terms, a particle or light ray in $\mathrm{C}$ will never escape this region, since its trajectory will inevitably hit the singularity. This is why $r=r_{\mathrm{s}}$ is called event horizon and the area with $r_{\mathrm{s}}>r>0$ and $T>0$ is the BH. ${ }^{\mathrm{a}}$

Initially, BHs were thought to be only a mathematical curiosity, but during the 1960s some physicists suggested that they could form with the collapse of massive stars (e.g. Ref. 2). To date, we know that BHs play an important role in many astrophysical scenarios. For instance, they are the main characters during TDEs.

a The symmetric region of the $\mathrm{BH}$ with respect to the origin is called white hole, and the origin which connects these two regions would be the wormhole. However the description of these objects is beyond the goal of this paper. 
Let us assume to have a star of mass $M_{*}$ and radius $R_{*}$, on a parabolic orbit around a $\mathrm{BH}$ of mass $M_{\mathrm{h}}$. For the sake of simplicity, the star interacts with the hole only at the pericenter, $r_{\mathrm{p}}$, of the orbit, that is the distance of closest approach between the star and the BH. If the star wanders too close to the hole, it gets tidally disrupted because the star self-gravity is overwhelmed by the BH tides. The minimum approach that the star can have without being destroyed is called tidal radius, $r_{\mathrm{t}}$, and reads ${ }^{3}$

$$
r_{\mathrm{t}}=R_{*}\left(\frac{M_{\mathrm{h}}}{M_{*}}\right)^{1 / 3} .
$$

So the disruption happens when $r_{\mathrm{p}} \leq r_{\mathrm{t}}$. If we introduce the dimensionless parameter $\beta \doteq r_{\mathrm{t}} / r_{\mathrm{p}}$, we have that the TDE occurs when $\beta$ varies in the range

$$
1<\beta \leq \beta_{\max }
$$

where $\beta_{\max }=r_{\mathrm{t}} / r_{\mathrm{s}}, r_{\mathrm{s}}$ being the Schwarzschild radius of the $\mathrm{BH}$, since, to see the TDE, we do not want the star to cross the event horizon. After the disruption, around half of the star escapes on hyperbolic orbits, the other half circularizes and forms a disc around the hole, that accretes material onto the central object. The accretion causes an accompanying electromagnetic flare, very luminous, and the lightcurve is consistent ${ }^{\mathrm{b}}$ with a $t^{-5 / 3}$ law for long times. $\frac{5}{5}$ So far, around 50 TDEs have been observed, and they have been detected in X-rays, $\gamma$-rays, radio and optical.

TDEs are also expected to emit GW bursts (e.g. Ref. 6). GWs are perturbations of the spacetime metric emitted by accelerated masses that produce a time varying mass quadrupole. ${ }^{7}$ Indeed, during TDEs, we have the realization of this scenario and consequently the production of a gravitational signal. Assuming that the star is a point-mass particle in a Keplerian motion around the hole, the GW strain, $h$, will be 8

$$
h \approx \beta \frac{r_{\mathrm{s} *} r_{\mathrm{gh}}}{d r_{\mathrm{t}}},
$$

where $r_{\mathrm{s}}$ is the Schwarzschild radius of the star, $r_{\mathrm{gh}}$ is the gravitational radius of the hole (half of $r_{\mathrm{s}}$ ) and $d$ is the distance from the source. Moreover, we expect the GW signal to have a frequency which is similar to the Keplerian one

$$
f \approx \frac{\beta^{3 / 2}}{2 \pi}\left(\frac{G M_{\mathrm{h}}}{r_{\mathrm{t}}^{3}}\right)^{1 / 2} .
$$

In the light of this, how can we relate TDEs with the detection of IMBHs? We need to consider TDEs of white dwarfs (WDs), since in this case we not only have a luminous electromagnetic event, but also a GW signal strong enough to be revealed by the new generation of GW interferometer, the Laser Interferometer

\footnotetext{
${ }^{\mathrm{b}} \mathrm{A}$ recent study ${ }^{4}$ shows that these lightcurves present different behaviors in different bands, however a more detailed discussion of TDEs is not relevant for this paper.
} 
Space Antenna (LISA) $\frac{9}{}$ Indeed, WDs are highly dense, small stars, with mass comparable to the one of the Sun. A typical relation that holds for the mass-radius of a WD is 10

$$
R_{*}=10^{-2} R_{\odot}\left(\frac{M_{\odot}}{M_{*}}\right)^{1 / 3},
$$

where $R_{\odot}$ and $M_{\odot}$ are the radius and the mass of the Sun, respectively. If we are interesting in studying WD TDEs, requiring

$$
r_{\mathrm{t}}>r_{\mathrm{s}}
$$

we find that the upper limit to the mass of the $\mathrm{BH}$ is $10^{5} M_{\odot}$, which implies that a reasonable mass range for $\mathrm{BHs}$ involved in this type of TDEs is $10^{3} M_{\odot}-10^{5} M_{\odot}$. The GW signal expected from this kind of disruption, assuming, e.g. to have a $10^{5} M_{\odot} \mathrm{BH}, \beta=1$ and that the source is in the nearest cluster of galaxies with respect to us (Virgo cluster, $20 \mathrm{Mpc}$ ), would have a strain and a frequency given by (Eqs. (8) and (9))

$$
\begin{aligned}
& h \approx 10^{-21}, \\
& f \approx 10^{-2}-10^{-1} \mathrm{~Hz} .
\end{aligned}
$$

From this simple estimates, we see that the signal is in the frequency range visible to LISA $\left(10^{-4} \mathrm{~Hz}, 1 \mathrm{~Hz}\right)$ and it is high enough to be detected when the interferometer will be launched in space.

So far, that are no solid clues on the existence of IMBHs. However, a recent work ${ }^{11}$ claimed to have observed a TDE with an electromagnetic signature that strongly suggests the presence of such an object. This underlines the importance that GWs will have in this field. Indeed, when it will be possible to see TDEs not only through the electromagnetic signal but also through GWs, we will be able to find and also confirm IMBHs. So, thanks to the help of LISA in the study of WD TDEs, it will be finally possible to reveal these objects that have been so much elusive to this date.

\section{References}

1. K. Schwarzschild, Über das Gravitationsfeld eines Massenpunktex nach der Einsteinschen Theorie, Sitzungsberichte der Königlich Preußischen Akademie der Wissenschaften (Berlin, 1916), pp. 189-196.

2. R. Penrose, Nuovo Cim. Riv. Serie 1 (1969) 252.

3. M. J. Rees, Nature 333 (1988) 523.

4. G. Lodato and E. M. Rossi, MNRAS 410 (2011) 359.

5. E. S. Phinney, Manifestations of a massive black hole in the galactic center, in The Center of the Galaxy, ed. M. Morris, IAU Symposium, Vol. 136 (Kluwer Academic Publishers, 1989), p. 543.

6. S. Kobayashi, P. Laguna, E. S. Phinney and P. Mészáros, 615 (2004) 855.

7. A. Einstein, Üner Gravitationswellen, Sitzungsberichte der Königlich Preußischen Akademie der Wissenschaften (Berlin, 1918), pp. 154-167. 
8. K. S. Thorne, Probing black holes and relativistic stars with gravitational waves, in Black Holes and Relativistic Stars, Proceedings of a Conference in Memory of S. Chandrasekhar, ed. R. M. Wald (University of Chicago Press, Chicago, 1998), pp. $41-78$.

9. P. Amaro-Seoane et al., arXiv:1702.00786.

10. S. L. Shapiro and S. A. Teukolsky, Black Holes, White Dwarfs, and Neutron Stars: The Physics of Compact Objects (Wiley-VCH, 1983).

11. D. Lin, J. Strader, E. R. Carrasco, D. Page, A. J. Romanowsky, J. Homan, J. A. Irwin, R. A. Remillard, O. Godet and N. A. Webb, Nat. Astron. 2 (2018) 656. 